\title{
Quantum Radiation Properties of Dirac Particles in General Nonstationary Black Holes
}

\author{
Jia-Chen Hua ${ }^{1,2}$ and Yong-Chang Huang ${ }^{2,3,4}$ \\ ${ }^{1}$ Department of Physics, University of Houston, Houston, TX 77204, USA \\ ${ }^{2}$ Institute of Theoretical Physics, Beijing University of Technology, Beijing 100124, China \\ ${ }^{3}$ Kavli Institute for Theoretical Physics, Chinese Academy of Sciences, Beijing 100190, China \\ ${ }^{4}$ CCAST (World Lab.), P.O. Box 8730, Beijing 100080, China
}

Correspondence should be addressed to Jia-Chen Hua; theorphysi@hotmail.com

Received 22 July 2013; Accepted 4 November 2013; Published 29 January 2014

Academic Editor: Frederik G. Scholtz

Copyright (C) 2014 J.-C. Hua and Y.-C. Huang. This is an open access article distributed under the Creative Commons Attribution License, which permits unrestricted use, distribution, and reproduction in any medium, provided the original work is properly cited. The publication of this article was funded by SCOAP S $^{3}$

Quantum radiation properties of Dirac particles in general nonstationary black holes in the general case are investigated by both using the method of generalized tortoise coordinate transformation and considering simultaneously the asymptotic behaviors of the first-order and second-order forms of Dirac equation near the event horizon. It is generally shown that the temperature and the shape of the event horizon of this kind of black holes depend on both the time and different angles. Further, we give a general expression of the new extra coupling effect in thermal radiation spectrum of Dirac particles which is absent from the thermal radiation spectrum of scalar particles. Also, we reveal a relationship that is ignored before between thermal radiation and nonthermal radiation in the case of scalar particles, which is that the chemical potential in thermal radiation spectrum is equal to the highest energy of the negative energy state of scalar particles in nonthermal radiation for general nonstationary black holes.

\section{Introductions}

Hawking's original discovery of quantum thermal radiation of black holes has been extensively studied in the fourth quarter of the last century $[1,2]$. An important subject in black hole physics is to reveal the thermal and nonthermal properties of various black holes. The last few decades had witnessed much progress in investigating the thermal radiation and nonthermal radiation of scalar fields or Dirac particles in many different kinds of black holes. Nevertheless, most of these researches had concentrated on static or stationary concrete black holes (e.g., [3-5]), since the original derivation of black hole evaporation involves gravitational collapse and is technically rather complicated $[1,2]$. It is, however, possible to understand the particle emissions within the approaches that do not depend heavily on the gravitational collapse itself. Thus, in 1976, Damour and Ruffini presented a treatment in which the gravitational field is independent of time [6]. In their approach the particle emission arises directly from a quantum mechanical barrier penetration across the event horizon of black holes. Sannan improved their method and obtained the probability distributions of both bosons and fermions emitted by black holes [7]. Then in the 1990s, [810] made further improvements in these parts and calculated the location of the event horizon and the temperature of nonstationary black holes simultaneously by using the method of generalized tortoise coordinate transformation and obtained the Hawking thermal spectrum. [8-10] Results of are consistent with those obtained by calculating the vacuum expectation values of the renormalized energymomentum tensors [11, 12] on some spherically symmetric nonstationary black holes. Therefore, one can obtain the event horizon equation, Hawking temperature, and thermal radiation spectrum of nonstationary black holes more conveniently and more exactly via the method of generalized tortoise coordinate transformation [13-15].

However, it is very difficult to deal with the quantum thermal effect of Dirac particles in nonstationary black 
holes. The difficulty mainly lies in the nonseparability of variables for Chandrasekhar-Dirac equations $[16,17]$ in the nonstationary axisymmetric and more general spacetime. Recently, [18-21] have suggested that considering simultaneously the asymptotic behaviors of the first-order and secondorder forms of Dirac equation near the event horizon would overcome this difficult problem. In [18-21], under the generalized tortoise coordinate transformation, each second order equation induced from Chandrasekhar-Dirac equation takes the standard form of wave equation near the event horizon to which separation of variables is possible. The location and the temperature of the event horizon are just the same as those obtained in the case of the thermal radiation of scalar particles in some nonstationary spacetime. Moreover, a kind of new term, representing a new extra spin-rotation coupling effect in [18-20] and a new extra spin-acceleration coupling effect in [21], appears in the Fermionic spectrum of Dirac particles. This kind of new term is absent from the Bosonic spectrum of scalar particles. However, these researches were restricted to concrete black holes and did not notice the relation between thermal radiation and nonthermal radiation in the case of scalar particles.

References [22-24] investigated thermodynamics of black holes in lovelock gravity and in AdS/dS spaces. One of the key issues of these researches is the calculation of the Hawking temperature. By using the method of generalized tortoise coordinate transformation, our paper may provide an alternative and convenient way to obtain the Hawking temperature in these researches.

The main purposes of this paper are to investigate the thermal and nonthermal radiation of Dirac particles in general nonstationary black holes and the relation between the two kinds of radiation in the case of scalar particles and give a general expression of the new extra coupling effect in thermal radiation spectrum of Dirac particles. Section 2 calculates the location of event horizon, the Hawking temperature, and the thermal radiation spectrum of Dirac particles in detail by using the method of generalized tortoise coordinate transformation [8-10,13-15] and considering simultaneously the asymptotic behaviors of the first-order and second-order forms of Dirac equation near the event horizon [18-21]. In Section 3, we formulate the highest energy of negative energy state of Dirac particles in nonthermal radiation by using the methods and the conclusions of [25-31]. Section 4 is the discussion. In this section, we analyse the thermal radiation spectrum of Dirac particles in general nonstationary black holes, give the general expression of the new extra coupling effect, and discuss the relationship between thermal radiation and nonthermal radiation of black holes in the case of scalar particles. The last section is summary and conclusion.

\section{Calculation of the Thermal Radiation in Detail}

The square of infinitesimal line element of the most general spacetime can be written as

$$
d s^{2}=g_{\mu \nu} d x^{\mu} d x^{\nu}
$$

where we take the advanced Eddington-Finkelstein coordinates $\left(x^{0}=v, x^{1}=r, x^{2}=\theta\right.$, and $\left.x^{3}=\varphi\right)$ and make the conventions that all indices of Latin letters $j, k=0,2,3$ and all indices of Greek letters $\mu, \nu=0,1,2,3$.

The surface equation of event horizon can be written as $F(v, r, \theta, \varphi)=0$, where $r_{H}=r_{H}(v, \theta, \varphi)$, which should satisfy null surface condition

$$
g^{\mu \nu} \frac{\partial F}{\partial x^{\mu}} \frac{\partial F}{\partial x^{\nu}}=0 .
$$

From the previous equation, we can obtain

$$
g^{11}-2 g^{1 j} r_{, j}+g^{j k} r_{, j} r_{, k}=0
$$

$r_{H}$ is the location of event horizon and depends on the time and different angles.

To write out the explicit form of Dirac equation in the Newman-Penrose (NP) formalism [32], we establish the following complex null-tetrad system $\left\{l^{\mu}, n^{\mu}, m^{\mu}, \bar{m}^{\mu}\right\}$ at each point in a 4-dimensional spacetime, where $l^{\mu}$ and $n^{\mu}$ are a pair of standard real null vectors such that $n^{\mu} l_{\mu}=1, m^{\mu}$ is the complex null vector, and the fourth vector $\bar{m}^{\mu}$ is just the complex conjugate of $m^{\mu}$. The NP tetrad satisfies the orthogonal conditions

$$
\begin{aligned}
& l_{\mu} l^{\mu}=n_{\mu} n^{\mu}=m_{\mu} m^{\mu}=\bar{m}_{\mu} \bar{m}^{\mu}=0, \\
& l_{\mu} n^{\mu}=-m_{\mu} \bar{m}^{\mu}=1, \\
& l_{\mu} m^{\mu}=l_{\mu} \bar{m}^{\mu}=n_{\mu} m^{\mu}=n_{\mu} \bar{m}^{\mu}=0, \\
& g^{\mu \nu}=l^{\mu} n^{v}+n^{\mu} l^{\nu}-m^{\mu} \bar{m}^{v}-\bar{m}^{\mu} m^{\nu}, \\
& g_{\mu \nu}=l_{\mu} n_{\nu}+n_{\mu} l_{v}-m_{\mu} \bar{m}_{\nu}-\bar{m}_{\mu} m_{v},
\end{aligned}
$$

and then we obtain the corresponding directional derivatives

$$
\begin{aligned}
& D=l^{\mu} \frac{\partial}{\partial x^{\mu}}, \\
& \Delta=n^{\mu} \frac{\partial}{\partial x^{\mu}}, \\
& \delta=m^{\mu} \frac{\partial}{\partial x^{\mu}}, \\
& \bar{\delta}=\bar{m}^{\mu} \frac{\partial}{\partial x^{\mu}} .
\end{aligned}
$$

The dynamical behavior of spin-1/2 particles in curved spacetime is described by the four coupled ChandrasekharDirac equations $[16,17]$ expressed in the Newman-Penrose formalism as follows:

$$
\begin{aligned}
& \left(D+\varepsilon-\rho+i e A_{\mu} l^{\mu}\right) F_{1}+\left(\bar{\delta}+\pi-\alpha+i e A_{\mu} \bar{m}^{\mu}\right) F_{2} \\
& -\frac{1}{\sqrt{2}} i \mu_{0} G_{1}=0,
\end{aligned}
$$




$$
\begin{aligned}
& \left(\Delta+\mu-\gamma+i e A_{\mu} n^{\mu}\right) F_{2}+\left(\delta+\beta-\tau+i e A_{\mu} m^{\mu}\right) F_{1} \\
& \quad-\frac{1}{\sqrt{2}} i \mu_{0} G_{2}=0, \\
& \left(D+\bar{\varepsilon}-\bar{\rho}+i e A_{\mu} l^{\mu}\right) G_{2}-\left(\delta+\bar{\pi}-\bar{\alpha}+i e A_{\mu} m^{\mu}\right) G_{1} \\
& \quad-\frac{1}{\sqrt{2}} i \mu_{0} F_{2}=0, \\
& \left(\Delta+\bar{\mu}-\bar{\gamma}+i e A_{\mu} \eta^{\mu}\right) G_{1}-\left(\bar{\delta}+\bar{\beta}-\bar{\tau}+i e A_{\mu} \bar{m}^{\mu}\right) G_{2} \\
& \quad-\frac{1}{\sqrt{2}} i \mu_{0} F_{1}=0,
\end{aligned}
$$

where $\mu_{0}$ and $e$ are the mass and charge of the Dirac particles, respectively. $F_{1}, F_{2}, G_{1}$, and $G_{2}$ are the four components of Dirac spinor in the Newman-Penrose formalism. $\varepsilon, \rho, \pi, \alpha, \mu, \gamma, \beta$, and $\tau$ are spin coefficients introduced by Newman and Penrose [32], which satisfy

$$
\begin{gathered}
\varepsilon=\frac{1}{2}\left(l_{\mu ; \nu} n^{\mu} l^{\nu}-m_{\mu ; \nu} \bar{m}^{\mu} l^{\nu}\right), \\
\rho=l_{\mu ; \nu} m^{\mu} \bar{m}^{\nu}, \\
\pi=-n_{\mu ; \nu} \bar{m}^{\mu} l^{\nu}, \\
\alpha=\frac{1}{2}\left(l_{\mu ; \nu} n^{\mu} \bar{m}^{\nu}-m_{\mu ; \nu} \bar{m}^{\mu} \bar{m}^{\nu}\right), \\
\mu=-n_{\mu ; \nu} \bar{m}^{\mu} m^{\nu}, \\
\gamma=\frac{1}{2}\left(l_{\mu ; \nu} n^{\mu} n^{\nu}-m_{\mu ; \nu} \bar{m}^{\mu} n^{\nu}\right), \\
\beta=\frac{1}{2}\left(l_{\mu ; \nu} n^{\mu} m^{\nu}-m_{\mu ; \nu} \bar{m}^{\mu} m^{\nu}\right), \\
\tau=l_{\mu ; \nu} m^{\mu} n^{\nu},
\end{gathered}
$$

and $\bar{\varepsilon}, \bar{\rho}, \bar{\pi}, \bar{\alpha}, \bar{\mu}, \bar{\gamma}, \bar{\beta}$, and $\bar{\tau}$ are complex conjugates of $\varepsilon, \rho, \pi, \alpha, \mu, \gamma, \beta$, and $\tau$.

To investigate the thermal radiation of spin-1/2 particles, we need to deal with the behavior of the second order Dirac equations near the event horizon. It is consistent to consider the asymptotic behavior of the first-order and second-order Dirac equations in the meantime because the four-component Dirac spinors should satisfy both of them. By substituting (6) and (7) into (8) and (9), one can obtain the second order form of Dirac equations for $\left(F_{1}, F_{2}\right)$ components as follows:

$$
\begin{aligned}
- & 2\left(\Delta+\bar{\mu}-\bar{\gamma}+i e A_{\mu} n^{\mu}\right) \\
& \times\left[\left(D+\varepsilon-\rho+i e A_{\mu} l^{\mu}\right) F_{1}+\left(\bar{\delta}+\pi-\alpha+i e A_{\mu} \bar{m}^{\mu}\right) F_{2}\right] \\
& +2\left(\bar{\delta}+\bar{\beta}-\bar{\tau}+i e A_{\mu} \bar{m}^{\mu}\right) \\
& \times\left[\left(\Delta+\mu-\gamma+i e A_{\mu} n^{\mu}\right) F_{2}+\left(\delta+\beta-\tau+i e A_{\mu} m^{\mu}\right) F_{1}\right] \\
& -\mu_{0}^{2} F_{1}=0,
\end{aligned}
$$

$$
\begin{aligned}
& -2\left(D+\bar{\varepsilon}-\bar{\rho}+i e A_{\mu} l^{\mu}\right) \\
& \quad \times\left[\left(\Delta+\mu-\gamma+i e A_{\mu} n^{\mu}\right) F_{2}+\left(\delta+\beta-\tau+i e A_{\mu} m^{\mu}\right) F_{1}\right] \\
& +2\left(\delta+\bar{\pi}-\bar{\alpha}+i e A_{\mu} m^{\mu}\right) \\
& \quad \times\left[\left(D+\varepsilon-\rho+i e A_{\mu} l^{\mu}\right) F_{1}+\left(\bar{\delta}+\pi-\alpha+i e A_{\mu} \bar{m}^{\mu}\right) F_{2}\right] \\
& -\mu_{0}^{2} F_{2}=0 .
\end{aligned}
$$

Introducing the generalized tortoise coordinate transformation $[6-10,13-15,18-21,33]$

$$
\begin{gathered}
r_{*}=r+\frac{1}{2 \kappa} \ln \left[r-r_{H}(v, \theta, \varphi)\right], \\
v_{*}=v-v_{0}, \\
\theta_{*}=\theta-\theta_{0}, \\
\varphi_{*}=\varphi-\varphi_{0}, \\
\frac{\partial}{\partial r}=\frac{\partial}{\partial r_{*}}+\frac{1}{2 \kappa\left(r-r_{H}\right)} \frac{\partial}{\partial r_{*}}, \\
\frac{\partial}{\partial x^{j}}=\frac{\partial}{\partial x_{*}^{j}}-\frac{r_{H, j}}{2 \kappa\left(r-r_{H}\right)} \frac{\partial}{\partial r_{*}},
\end{gathered}
$$

along with

$$
\begin{gathered}
\frac{\partial^{2}}{\partial r^{2}}=\frac{\left[2 \kappa\left(r-r_{H}\right)+1\right]^{2}}{\left[2 \kappa\left(r-r_{H}\right)\right]^{2}} \frac{\partial^{2}}{\partial r_{*}^{2}}-\frac{1}{2 \kappa\left(r-r_{H}\right)^{2}} \frac{\partial}{\partial r_{*}}, \\
\frac{\partial^{2}}{\partial r \partial x^{j}}=\frac{2 \kappa\left(r-r_{H}\right)+1}{2 \kappa\left(r-r_{H}\right)} \frac{\partial^{2}}{\partial r_{*} \partial x_{*}^{j}} \\
+\frac{r_{H, j} 2 \kappa}{\left[2 \kappa\left(r-r_{H}\right)\right]^{2}} \frac{\partial}{\partial r_{*}}-\frac{r_{H, j}\left[2 \kappa\left(r-r_{H}\right)+1\right]}{\left[2 \kappa\left(r-r_{H}\right)\right]^{2}} \frac{\partial^{2}}{\partial r_{*}^{2}}, \\
\frac{\partial^{2}}{\partial x^{j} \partial x^{k}}=\frac{\partial^{2}}{\partial x_{*}^{j} \partial x_{*}^{k}-\frac{r_{H, j}}{2 \kappa\left(r-r_{H}\right)} \frac{\partial^{2}}{\partial r_{*} \partial x_{*}^{k}}} \\
-\frac{r_{H, k}}{2 \kappa\left(r-r_{H}\right)} \frac{\partial^{2}}{\partial r_{*} \partial x_{*}^{j}}+\frac{r_{H, j} r_{H, k}}{\left[2 \kappa\left(r-r_{H}\right)\right]^{2}} \frac{\partial^{2}}{\partial r_{*}^{2}} \\
-\frac{2 \kappa\left(r-r_{H}\right) r_{H, j k}+2 \kappa r_{H, j} r_{H, k}}{\left[2 \kappa\left(r-r_{H}\right)\right]^{2}},
\end{gathered}
$$

where $v_{0}, \theta_{0}$, and $\varphi_{0}$ are parameters under the tortoise transformation and $\kappa \equiv \kappa\left(v_{0}, \theta_{0}, \varphi_{0}\right)$ is an adjustable parameter that depends on time and angular coordinates.

The methods of generalized tortoise coordinate transformation can give simultaneously the exact values of the location and the temperature of the event horizon of nonstationary black holes. Basically, this method reduces KleinGordon or Dirac equation in a known black hole background to a standard wave equation outside and near the 
event horizon by generalizing the common tortoise-type coordinate $r_{*}=r+(1 / 2 \kappa) \ln \left(r-r_{H}\right)$ in a static or stationary spacetime $[6,7]$ (where $\kappa$ is the surface gravity of the studied event horizon) to a similar form in a nonstatic or nonstationary spacetime and by allowing the location of the event horizon $r_{H}$ to be a function of the advanced time $v=t+r_{*}$ and the angles $\theta$ and $\varphi$. The tortoise coordinates only describe the spacetime outside the event horizon and in this condition, $r_{*}$ tends to positive infinity when approaching to the infinite point and $r_{*}$ tends to negative infinity at the event horizon. In fact, this felicitously describes the spacetime outside the event horizon that we want to investigate, in which the tortoise coordinates do not result in the singularities (when we try to get the KleinGordon equation near the event horizon, we can take the limit of $r_{*}$ tending to negative infinity, i.e., $r \rightarrow r_{H}$; then we obtain the definitely finite form of the Klein-Gordon equation near the event horizon). And also, for the spacetime inside the event horizon, we can extend the outgoing wave function by analytic continuation into the event horizon through the lower-half complex $r$-plane (see the paragraph before (29) for details).

Applying the generalized tortoise coordinate transformation to (6)-(9) and taking the limit of $r \rightarrow r_{H}$ (here and hereafter, $r \rightarrow r_{H}$ represents $v \rightarrow v_{0}, \theta \rightarrow \theta_{0}, \varphi \rightarrow \varphi_{0}$, and $\left.r \rightarrow r_{H}\left(v_{0}, \theta_{0}, \varphi_{0}\right)\right)$, we can get

$$
\begin{aligned}
& \frac{\partial F_{1}}{\partial r_{*}}=\left.\frac{\bar{m}^{1}-\bar{m}^{j} r_{H, j}}{l^{j} r_{H, j}-l^{1}}\right|_{r \rightarrow r_{H}} \frac{\partial F_{2}}{\partial r_{*}}, \\
& \frac{\partial F_{2}}{\partial r_{*}}=\left.\frac{m^{1}-m^{j} r_{H, j}}{n^{j} r_{H, j}-n^{1}}\right|_{r \rightarrow r_{H}} \frac{\partial F_{1}}{\partial r_{*}}, \\
& \frac{\partial G_{2}}{\partial r_{*}}=\left.\frac{m^{1}-m^{j} r_{H, j}}{l^{1}-l^{j} r_{H, j}}\right|_{r \rightarrow r_{H}} \frac{\partial G_{1}}{\partial r_{*}}, \\
& \frac{\partial G_{1}}{\partial r_{*}}=\left.\frac{\bar{m}^{1}-\bar{m}^{j} r_{H, j}}{n^{1}-n^{j} r_{H, j}}\right|_{r \rightarrow r_{H}} \frac{\partial G_{2}}{\partial r_{*}} .
\end{aligned}
$$

Then applying the generalized tortoise coordinate transformation to (11), via some arrangement, multiplying $(2 \kappa(r-$ $\left.\left.r_{H}\right)\right) /\left(g^{01}\left[2 \kappa\left(r-r_{H}\right)+1\right]-g^{0 j} r_{H, j}\right)$ to both sides of the two second order equations for the coefficient of $\partial^{2} F_{1} / \partial r_{*} \partial v_{*}$ and $\partial^{2} F_{2} / \partial r_{*} \partial v_{*}$ to be $2[8-10,13-15,18-21]$, and finally taking the limit of $r \rightarrow r_{H}$ and substituting (14) into the two equations, we can obtain the following united form of them:

$$
I \frac{\partial^{2} \Psi}{\partial r_{*}^{2}}+2 \frac{\partial^{2} \Psi}{\partial r_{*} \partial \nu_{*}}+B \frac{\partial^{2} \Psi}{\partial r_{*} \partial \theta_{*}}+C \frac{\partial^{2} \Psi}{\partial r_{*} \partial \varphi_{*}}+A_{*} \frac{\partial \Psi}{\partial r_{*}}=0
$$

where

$$
\begin{aligned}
& I=\lim _{r \rightarrow r_{H}}\left(g^{11}\left[2 \kappa\left(r-r_{H}\right)+1\right]^{2}-2 g^{1 j} r_{H, j}\right. \\
& \left.\times\left[2 \kappa\left(r-r_{H}\right)+1\right]+g^{j k} r_{H, j} r_{H, k}\right) \\
& \times\left(2 \kappa\left(r-r_{H}\right)\left[g^{01}\left[2 \kappa\left(r-r_{H}\right)+1\right]-g^{0 j} r_{H, j}\right]\right)^{-1}, \\
& B=\left.2 \frac{g^{12}-g^{j 2} r_{H, j}}{g^{01}-g^{0 j} r_{H, j}}\right|_{r \rightarrow r_{H}}, \\
& C=\left.2 \frac{g^{13}-g^{j 3} r_{H, j}}{g^{01}-g^{0 j} r_{H, j}}\right|_{r \rightarrow r_{H}}, \\
& A_{*}=2 i\left(C_{0}+C_{1}\right)+C_{2}+C_{3 *}+C_{4} \text {, } \\
& C_{0}=\left.e \frac{A_{\mu} g^{\mu 1}-A_{\mu} g^{\mu j} r_{H, j}}{g^{01}-g^{0 j} r_{H, j}}\right|_{r \rightarrow r_{H}}, \\
& C_{3 *}=-2 \kappa\left(\left(g^{11}-2 g^{1 j} r_{H, j}+g^{j k} r_{H, j} r_{H, k}\right)\right. \\
& \times\left(2 \kappa ( r - r _ { H } ) \left[g^{01}\left[2 \kappa\left(r-r_{H}\right)+1\right]\right.\right. \\
& \left.\left.\left.-g^{0 j} r_{H, j}\right]\right)^{-1}\right)\left.\right|_{r \rightarrow r_{H}} \\
& C_{4}=\left.\frac{-g^{j k} r_{H, j k}}{g^{01}-g^{0 j} r_{H, j}}\right|_{r \rightarrow r_{H}}, \\
& C_{2}=\left.\frac{1}{g^{01}-g^{0 j} r_{H, j}}\right|_{r \rightarrow r_{H}} \\
& \times\left\{\left\{2 n^{\mu} l_{, \mu}^{1}-2(\beta-\tau) \bar{m}^{1}\right.\right. \\
& -2(\bar{\beta}-\bar{\tau}) m^{1}+[(\varepsilon-\rho)+(\bar{\varepsilon}-\bar{\rho})] n^{1} \\
& \left.+[(\bar{\mu}-\bar{\gamma})+(\mu-\gamma)] l^{1}-\left(\bar{m}^{\mu} m_{, \mu}^{1}+m^{\mu} \bar{m}_{, \mu}^{1}\right)\right\} \\
& -\left\{2 n^{\mu} l_{, \mu}^{j}-2(\beta-\tau) \bar{m}^{j}-2(\bar{\beta}-\bar{\tau}) m^{j}\right. \\
& +[(\varepsilon-\rho)+(\bar{\varepsilon}-\bar{\rho})] n^{j} \\
& +[(\bar{\mu}-\bar{\gamma})+(\mu-\gamma)] l^{j} \\
& \left.\left.-\left(\bar{m}^{\mu} m_{, \mu}^{j}+m^{\mu} \bar{m}_{, \mu}^{j}\right)\right\} r_{H, j}\right\}\left.\right|_{r \rightarrow r_{H}} \\
& +\left.\frac{1}{\left(g^{01}-g^{0 j} r_{H, j}\right)\left(n^{j} r_{H, j}-n^{1}\right)}\right|_{r \rightarrow r_{H}} \\
& \times\left\{\left\{\left[n^{\mu} \bar{m}_{, \mu}^{1}-\bar{m}^{\mu} n_{, \mu}^{1}+(\pi-\alpha) n^{1}\right.\right.\right. \\
& \left.+(\bar{\mu}-\bar{\gamma}) \bar{m}^{1}-(\mu-\gamma) \bar{m}^{1}-(\bar{\beta}-\bar{\tau}) n^{1}\right] \\
& -\left[n^{\mu} \bar{m}_{, \mu}^{j}-\bar{m}^{\mu} n_{, \mu}^{j}+(\pi-\alpha) n^{j}+(\bar{\mu}-\bar{\gamma}) \bar{m}^{j}\right.
\end{aligned}
$$




$$
\begin{aligned}
& \left.\left.-(\mu-\gamma) \bar{m}^{j}-(\bar{\beta}-\bar{\tau}) n^{j}\right] r_{H, j}\right\} \quad \text { for } \Psi=F_{1} \text {, and } \\
& \times\left(m^{1}-m^{j} r_{H, j}\right) \\
& +\left\{\left[n^{\mu} m_{, \mu}^{1}-m^{\mu} n_{, \mu}^{1}+(\bar{\pi}-\bar{\alpha}) n^{1}+(\mu-\gamma) m^{1}\right.\right. \\
& \left.-(\bar{\mu}-\bar{\gamma}) m^{1}-(\beta-\tau) n^{1}\right] \\
& -\left[n^{\mu} m_{, \mu}^{j}-m^{\mu} n_{, \mu}^{j}+(\bar{\pi}-\bar{\alpha}) n^{j}\right. \\
& +(\mu-\gamma) m^{j}-(\bar{\mu}-\bar{\gamma}) m^{j} \\
& \left.\left.\left.-(\beta-\tau) n^{j}\right] r_{H, j}\right\}\left(\bar{m}^{1}-\bar{m}^{j} r_{H, j}\right)\right\}\left.\right|_{r \rightarrow r_{H}} . \\
& C_{1}=\left.\frac{1}{g^{01}-g^{0 j} r_{H, j}}\right|_{r \rightarrow r_{H}} \\
& \times\left\{\left\{\frac{[(\bar{\mu}-\bar{\gamma})-(\mu-\gamma)] l^{1}}{2 i}+\frac{[(\varepsilon-\rho)-(\bar{\varepsilon}-\bar{\rho})] n^{1}}{2 i}\right.\right. \\
& \left.-\frac{\left(\bar{m}^{\mu} m_{, \mu}^{1}-m^{\mu} \bar{m}_{, \mu}^{1}\right)}{2 i}\right\} \\
& -\left\{\frac{[(\bar{\mu}-\bar{\gamma})-(\mu-\gamma)] l^{j}}{2 i}+\frac{[(\varepsilon-\rho)-(\bar{\varepsilon}-\bar{\rho})] n^{j}}{2 i}\right. \\
& \left.\left.-\frac{\left(\bar{m}^{\mu} m_{, \mu}^{j}-m^{\mu} \bar{m}_{, \mu}^{j}\right)}{2 i}\right\} r_{H, j}\right\}\left.\right|_{r \rightarrow r_{H}} \\
& +\left.\frac{1}{\left(g^{01}-g^{0 j} r_{H, j}\right)\left(n^{j} r_{H, j}-n^{1}\right)}\right|_{r \rightarrow r_{H}} \\
& \times \frac{1}{2 i}\left\{\left\{\left[n^{\mu} \bar{m}_{, \mu}^{1}-\bar{m}^{\mu} n_{, \mu}^{1}+(\pi-\alpha) n^{1}+(\bar{\mu}-\bar{\gamma}) \bar{m}^{1}\right.\right.\right. \\
& \left.-(\mu-\gamma) \bar{m}^{1}-(\bar{\beta}-\bar{\tau}) n^{1}\right] \\
& \text { - }\left[n^{\mu} \bar{m}_{, \mu}^{j}-\bar{m}^{\mu} n_{, \mu}^{j}+(\pi-\alpha) n^{j}+(\bar{\mu}-\bar{\gamma}) \bar{m}^{j}\right. \\
& \left.\left.-(\mu-\gamma) \bar{m}^{j}-(\bar{\beta}-\bar{\tau}) n^{j}\right] r_{H, j}\right\} \\
& \times\left(m^{1}-m^{j} r_{H, j}\right) \\
& -\left\{\left[n^{\mu} m_{, \mu}^{1}-m^{\mu} n_{, \mu}^{1}+(\bar{\pi}-\bar{\alpha}) n^{1}+(\mu-\gamma) m^{1}\right.\right. \\
& \left.\left.-(\bar{\mu}-\bar{\gamma}) m^{1}\right]-(\beta-\tau) n^{1}\right] \\
& -\left[n^{\mu} m_{, \mu}^{j}-m^{\mu} n_{, \mu}^{j}+(\bar{\pi}-\bar{\alpha}) n^{j}\right. \\
& +(\mu-\gamma) m^{j}-(\bar{\mu}-\bar{\gamma}) m^{j} \\
& \left.\left.-(\beta-\tau) n^{j}\right] r_{H, j}\right\} \\
& \left.\times\left(\bar{m}^{1}-\bar{m}^{j} r_{H, j}\right)\right\}\left.\right|_{r \rightarrow r_{H}}, \\
& C_{2}=\left.\frac{1}{g^{01}-g^{0 j} r_{H, j}}\right|_{r \rightarrow r_{H}} \\
& \times\left\{\left\{2 l^{\mu} n_{, \mu}^{1}-2(\pi-\alpha) m^{1}\right.\right. \\
& -2(\bar{\pi}-\bar{\alpha}) \bar{m}^{1}+[(\mu-\gamma)+(\bar{\mu}-\bar{\gamma})] l^{1} \\
& \left.+[(\bar{\varepsilon}-\bar{\rho})+(\varepsilon-\rho)] n^{1}-\left(m^{\mu} \bar{m}_{, \mu}^{1}+\bar{m}^{\mu} m_{, \mu}^{1}\right)\right\} \\
& -\left\{2 l^{\mu} n_{, \mu}^{j}-2(\pi-\alpha) m^{j}-2(\bar{\pi}-\bar{\alpha}) \bar{m}^{j}\right. \\
& +[(\mu-\gamma)+(\bar{\mu}-\bar{\gamma})] l^{j}+[(\bar{\varepsilon}-\bar{\rho})+(\varepsilon-\rho)] n^{j} \\
& \left.\left.-\left(m^{\mu} \bar{m}_{, \mu}^{j}+\bar{m}^{\mu} m_{, \mu}^{j}\right)\right\} r_{H, j}\right\}\left.\right|_{r \rightarrow r_{H}} \\
& +\left.\frac{1}{\left(g^{01}-g^{0 j} r_{H, j}\right)\left(l^{j} r_{H, j}-l^{1}\right)}\right|_{r \rightarrow r_{H}} \\
& \times\left\{\left\{\left[l^{\mu} m_{, \mu}^{1}-m^{\mu} l_{, \mu}^{1}\right.\right.\right. \\
& +(\beta-\tau) l^{1}+(\bar{\varepsilon}-\bar{\rho}) m^{1}-(\varepsilon-\rho) m^{1} \\
& \left.-(\bar{\pi}-\bar{\alpha}) l^{1}\right]-\left[l^{\mu} m_{, \mu}^{j}-m^{\mu} l_{, \mu}^{j}+(\beta-\tau) l^{j}\right. \\
& +(\bar{\varepsilon}-\bar{\rho}) m^{j}-(\varepsilon-\rho) m^{j} \\
& \left.\left.-(\bar{\pi}-\bar{\alpha}) l^{j}\right] r_{H, j}\right\} \\
& \times\left(\bar{m}^{1}-\bar{m}^{j} r_{H, j}\right) \\
& +\left\{\left[l^{\mu} \bar{m}_{, \mu}^{1}-\bar{m}^{\mu} l_{, \mu}^{1}+(\bar{\beta}-\bar{\tau}) l^{1}\right.\right. \\
& \left.+(\varepsilon-\rho) \bar{m}^{1}-(\bar{\varepsilon}-\bar{\rho}) \bar{m}^{1}-(\pi-\alpha) l^{1}\right] \\
& -\left[l^{\mu} \bar{m}_{, \mu}^{j}-\bar{m}^{\mu} l_{, \mu}^{j}+(\bar{\beta}-\bar{\tau}) l^{j}+(\varepsilon-\rho) \bar{m}^{j}\right. \\
& \left.\left.-(\bar{\varepsilon}-\bar{\rho}) \bar{m}^{j}-(\pi-\alpha) l^{j}\right] r_{H, j}\right\} \\
& \left.\times\left(m^{1}-m^{j} r_{H, j}\right)\right\}\left.\right|_{r \rightarrow r_{H}}, \\
& C_{1}=\left.\frac{1}{g^{01}-g^{0 j} r_{H, j}}\right|_{r \rightarrow r_{H}} \\
& \times\left\{\left\{\frac{[(\mu-\gamma)-(\bar{\mu}-\bar{\gamma})] l^{1}}{2 i}+\frac{[(\bar{\varepsilon}-\bar{\rho})-(\varepsilon-\rho)] n^{1}}{2 i}\right.\right. \\
& \left.-\frac{\left(m^{\mu} \bar{m}_{, \mu}^{1}-\bar{m}^{\mu} m_{, \mu}^{1}\right)}{2 i}\right\} \\
& -\left\{\frac{[(\mu-\gamma)-(\bar{\mu}-\bar{\gamma})] l^{j}}{2 i}+\frac{[(\bar{\varepsilon}-\bar{\rho})-(\varepsilon-\rho)] n^{j}}{2 i}\right.
\end{aligned}
$$




$$
\begin{gathered}
\left.\left.-\frac{\left(m^{\mu} \bar{m}_{, \mu}^{j}-\bar{m}^{\mu} m_{, \mu}^{j}\right)}{2 i}\right\} r_{H, j}\right\}\left.\right|_{r \rightarrow r_{H}} \\
+\left.\frac{1}{\left(g^{01}-g^{0 j} r_{H, j}\right)\left(l^{j} r_{H, j}-l^{1}\right)}\right|_{r \rightarrow r_{H}} \\
\times \frac{1}{2 i}\left\{\left\{\left[l^{\mu} m_{, \mu-m^{\mu} l_{, \mu}^{1}}^{1}+(\beta-\tau) l^{1}\right.\right.\right. \\
\left.+(\bar{\varepsilon}-\bar{\rho}) m^{1}-(\varepsilon-\rho) m^{1}-(\bar{\pi}-\bar{\alpha}) l^{1}\right] \\
-\left[l^{\mu} m_{, \mu}^{j}-m^{\mu} l_{, \mu}^{j}+(\beta-\tau) l^{j}+(\bar{\varepsilon}-\bar{\rho}) m^{j}\right. \\
\left.\left.-(\varepsilon-\rho) m^{j}-(\bar{\pi}-\bar{\alpha}) l^{j}\right] r_{H, j}\right\}\left(\bar{m}^{1}-\bar{m}^{j} r_{H, j}\right) \\
-\left\{\left[l^{\mu} \bar{m}_{, \mu}^{1}-\bar{m}^{\mu} l_{, \mu}^{1}+(\bar{\beta}-\bar{\tau}) l^{1}\right.\right. \\
\left.+(\varepsilon-\rho) \bar{m}^{1}-(\bar{\varepsilon}-\bar{\rho}) \bar{m}^{1}-(\pi-\alpha) l^{1}\right] \\
\left.\times\left(m^{1}-m^{j} r_{H, j}\right)\right\}\left.\right|_{r \rightarrow r_{H}} \\
-\left[l^{\mu} \bar{m}_{, \mu}^{j}-\bar{m}^{\mu} l_{, \mu}^{j}+(\bar{\beta}-\bar{\tau}) l^{j}+(\varepsilon-\rho) \bar{m}^{j}\right. \\
\left.\left.-(\bar{\varepsilon}-\bar{\rho}) \bar{m}^{j}-(\pi-\alpha) l^{j}\right] r_{H, j}\right\}
\end{gathered}
$$

for $\Psi=F_{2}$.

Here the numerator of $I$ is

$$
g^{11}-2 g^{1 j} r_{H, j}+\left.g^{j k} r_{H, j} r_{H, k}\right|_{r \rightarrow r_{H}} .
$$

One can see that this is the left side of (3), so the numerator approaches to zero, and the denominator also approaches to zero. Hence, $I$ is an indeterminate form of $0 / 0$ type. Using L'Hospital's Rule and adjusting $\kappa$ to let $I$ be 1 [8-10, 13-15, 18-21]; then (15) becomes the standard wave equation in flat spacetime consequently; that is,

$$
\begin{gathered}
I=\left(\left(\frac{\partial g^{11}}{\partial r}-2 \frac{\partial g^{1 j}}{\partial r} r_{H, j}+\frac{\partial g^{j k}}{\partial r} r_{H, j} r_{H, k}\right.\right. \\
\left.+2 \kappa\left[2 g^{11}-2 g^{0 j} r_{H, j}\right]\right) \\
\left.\times\left(2 \kappa\left[g^{01}-g^{0 j} r_{H, j}\right]\right)^{-1}\right)\left.\right|_{r \rightarrow r_{H}}
\end{gathered}
$$

$$
=1 \text {. }
$$

Thus, we can gain

$$
\kappa=\left.\frac{\left(\partial g^{11} / \partial r\right)+\left(\partial g^{j k} / \partial r\right) r_{H, j} r_{H, k}-2\left(\partial g^{j 1} / \partial r\right) r_{H, j}}{2\left[g^{01}-2 g^{11}+\left(2 g^{j 1}-g^{j 0}\right) r_{H, j}\right]}\right|_{r \rightarrow r_{H}},
$$

which is the "surface gravity" of event horizon.
By the same reason, $C_{3 *}$ is also an indeterminate form of $0 / 0$ type. According to the similar method we can obtain

$$
\begin{array}{r}
-2 \kappa \lim _{r \rightarrow r_{H}} \frac{g^{11}-2 g^{1 j} r_{H, j}+g^{j k} r_{H, j} r_{H, k}}{2 \kappa\left(r-r_{H}\right)\left[g^{01}\left[2 \kappa\left(r-r_{H}\right)+1\right]-g^{0 j} r_{H, j}\right]} \\
=-\left.2 \kappa\left[1-2 \frac{g^{11}-g^{j 1} r_{H, j}}{g^{01}-g^{0 j} r_{H, j}}\right]\right|_{r \rightarrow r_{H}} .
\end{array}
$$

Then the united form of second order Dirac equations (14) near the event horizon becomes the following form:

$$
\frac{\partial^{2} \Psi}{\partial r_{*}^{2}}+2 \frac{\partial^{2} \Psi}{\partial r_{*} \partial \nu_{*}}+B \frac{\partial^{2} \Psi}{\partial r_{*} \partial \theta_{*}}+C \frac{\partial^{2} \Psi}{\partial r_{*} \partial \varphi_{*}}+A \frac{\partial \Psi}{\partial r_{*}}=0
$$

where

$$
\begin{aligned}
& A=2 i\left(C_{0}+C_{1}\right)+C_{2}+C_{3}+C_{4}, \\
& C_{3}=-\left.2 \kappa\left[1-2 \frac{g^{11}-g^{j 1} r_{H, j}}{g^{01}-g^{0 j} r_{H, j}}\right]\right|_{r \rightarrow r_{H}} .
\end{aligned}
$$

Equation (22) can be viewed as a usual differential equation because all coefficients in it are regarded as finite constants. Thus, it can be treated by separating variables as $[10,13-15,18-$ 21]

$$
\Psi=R\left(r_{*}, v_{*}\right) \eta\left(v_{*}, \theta_{*}, \varphi_{*}\right) e^{-i \omega v_{*}+i k_{\theta} \theta_{*}+i k_{\varphi} \varphi_{*}},
$$

where $\eta\left(v_{*}, \theta_{*}, \varphi_{*}\right)$ is an arbitrary real function, $\omega$ is the energy of the Dirac particle which depends on tortoise coordinates, and $k_{\theta}$ and $k_{\varphi}$ are components of generalized momentum of Dirac particles. And we define $k_{\theta} \equiv P_{2} \equiv$ $\partial S / \partial \theta_{*}$ and $k_{\varphi} \equiv P_{3} \equiv \partial S / \partial \varphi_{*}$, where $S$ is the Hamiltonian main function of Dirac particles. When considering (22) near the event horizon, we are taking the limit of $r \rightarrow r_{H}$ such that the parameters $\omega, k_{\theta}$, and $k_{\varphi}$ in (24) tend to constants, which will simplify the following variable separation.

Substituting (24) into (22) after separating variables, one can gain two independent solutions

$$
\begin{aligned}
\Psi_{\text {in }}= & e^{1 / 2 \int\left[\xi-\lambda\left(v_{*}\right)\right] d v_{*}} \eta\left(v_{*}, \theta_{*}, \varphi_{*}\right) \\
& \times \exp \left(-i \omega v_{*}+i k_{\theta} \theta_{*}+i k_{\varphi} \varphi_{*}\right), \\
\Psi_{\text {out }}= & e^{1 / 2 \int\left[\xi-\lambda\left(v_{*}\right)\right] d v_{*}} \eta\left(v_{*}, \theta_{*}, \varphi_{*}\right) \\
& \times \exp \left(2 i \omega-A-i k_{\theta} B-i k_{\varphi} C-\xi\right) r_{*} \\
& \cdot \exp \left(-i \omega v_{*}+i k_{\theta} \theta_{*}+i k_{\varphi} \varphi_{*}\right),
\end{aligned}
$$

where $\xi$ is a constant in variable separation and $\lambda\left(v_{*}\right)$ is a function of the advanced time $v_{*}$ in variable separation, where $\lambda\left(v_{*}\right)=2\left(\left(\partial \eta / \partial v_{*}\right) / \eta\right)+B\left(\left(\partial \eta / \partial \theta_{*}\right) / \eta\right)+$ $C\left(\left(\partial \eta / \partial \varphi_{*}\right) / \eta\right)$ and $\xi=\left(2 T^{\prime}+\lambda\left(v_{*}\right) T\right) / T$ (we let $R\left(r_{*}, v_{*}\right)=$ $P\left(r_{*}\right) T\left(v_{*}\right)$ in variable separation). 
The radial components of the above two independent solutions are

$$
\begin{aligned}
& \psi_{\text {in }}=e^{1 / 2 \int\left[\xi-\lambda\left(v_{*}\right)\right] d v_{*}} \exp \left(-i \omega v_{*}\right), \\
& \psi_{\text {out }}=e^{1 / 2 \int\left[\xi-\lambda\left(v_{*}\right)\right] d v_{*}} \\
& \times \exp \left(2 i \omega-A-i k_{\theta} B-i k_{\varphi} C-\xi\right) r_{*} \cdot \exp \left(-i \omega v_{*}\right) \\
& =e^{1 / 2 \int\left[\xi-\lambda\left(v_{*}\right)\right] d v_{*}} e^{-i \omega v_{*}} \\
& \times e^{2 i\left(\omega-k_{\theta}(B / 2)-k_{\varphi}(C / 2)-(\operatorname{Im}(A) / 2)\right) r_{*}} e^{[-\xi-\operatorname{Re}(A)] r_{*}} .
\end{aligned}
$$

Because $\psi_{\text {out }}$ is not analytic at the event horizon, it needs extending by analytic continuation into the event horizon through the lower-half complex $r$-plane as $[6,7,34]$

$$
\left(r-r_{H}\right) \longrightarrow\left|r-r_{H}\right| e^{-i \pi}=\left(r_{H}-r\right) e^{-i \pi} .
$$

Hence

$$
\begin{aligned}
\widetilde{\psi}_{\text {out }}=[ & e^{1 / 2 \int\left[\xi-\lambda\left(v_{*}\right)\right] d v_{*}} e^{-i \omega v_{*}} \\
& \left.\times e^{2 i\left(\omega-k_{\theta}(B / 2)-k_{\varphi}(C / 2)-(\operatorname{Im}(A) / 2)\right) r_{*}} e^{[-\xi-\operatorname{Re}(A)] r_{*}}\right] \\
& \cdot e^{\pi / \kappa\left(\omega-k_{\theta}(B / 2)-k_{\varphi}(C / 2)-(\operatorname{Im}(A) / 2)\right)} \cdot e^{i \pi((\operatorname{Re}(A)+\xi) / 2 \kappa)} .
\end{aligned}
$$

From (28) and (30), we can obtain the relative scattering probability at the event horizon

$$
\left|\frac{\psi_{\text {out }}}{\widetilde{\psi}_{\text {out }}}\right|^{2}=e^{-2 \pi / \kappa\left(\omega-\omega_{0}\right)},
$$

where

$$
\omega_{0}=k_{\theta} \frac{B}{2}+k_{\varphi} \frac{C}{2}+\frac{\operatorname{Im}(A)}{2} .
$$

Following Damour and Ruffini [6] and Sannan [7], we can get the thermal radiation spectrum of Dirac particles (or scalar particles) from general nonstationary black holes

$$
N_{\omega}=\frac{1}{e^{\left(\omega-\omega_{0}\right) / k_{B} T} \pm 1}
$$

and the Hawking temperature

$$
T=\frac{\kappa}{2 \pi k_{B}}
$$

where $k_{B}$ is the Boltzmann constant and " \pm " correspond to fermion and boson, respectively. The temperature $T$ is

$$
\begin{aligned}
T & =\frac{\kappa}{2 \pi k_{B}} \\
& =\left.\frac{\left(\partial g^{11} / \partial r\right)+\left(\partial g^{j k} / \partial r\right) r_{H, j} r_{H, k}-2\left(\partial g^{j 1} / \partial r\right) r_{H, j}}{4 \pi k_{B}\left[g^{01}-2 g^{11}+\left(2 g^{j 1}-g^{j 0}\right) r_{H, j}\right]}\right|_{r \rightarrow r_{H}} .
\end{aligned}
$$

And the chemical potential is

$$
\begin{aligned}
\omega_{0}= & \left.k_{\theta} \frac{g^{12}-g^{j 2} r_{H, j}}{g^{01}-g^{0 j} r_{H, j}}\right|_{r \rightarrow r_{H}}+\left.k_{\varphi} \frac{g^{13}-g^{j 3} r_{H, j}}{g^{01}-g^{0 j} r_{H, j}}\right|_{r \rightarrow r_{H}} \\
& +\left.e \frac{A_{\mu} g^{\mu 1}-A_{\mu} g^{\mu j} r_{H, j}}{g^{01}-g^{0 j} r_{H, j}}\right|_{r \rightarrow r_{H}}+C_{1} .
\end{aligned}
$$

We know from (35) that $T$ depends on the time and different angles, so it is a distribution of temperatures. It is also interesting to find that the coefficient $C_{1}$ appears in the chemical potential, which may represent a particular energy term for Dirac particles. The physical meaning of $C_{1}$ will be discussed in Section 4.

\section{Research on Nonthermal Radiation of General Nonstationary Black Holes}

Now we use the methods and conclusions of [25-31] to investigate the nonthermal radiation.

Consider the Hamilton-Jacobi equation of moving particles

$$
g^{\mu \nu}\left(\frac{\partial S}{\partial x^{\mu}}+e A_{\mu}\right)\left(\frac{\partial S}{\partial x^{\nu}}+e A_{\nu}\right)+\mu_{0}^{2}=0 .
$$

Applying the generalized tortoise coordinate transformation to (37), via some simplifications, we let the solution of $\partial S / \partial r_{*}$ be real and define $\partial S / \partial v_{*} \equiv-\omega, \partial S / \partial \theta_{*} \equiv P_{2} \equiv$ $k_{\theta}, \partial S / \partial \varphi_{*} \equiv P_{3} \equiv k_{\varphi}$. Finally we can obtain the energy level distribution of the particles

$$
\omega \geq \omega^{+}, \quad \omega \leq \omega^{-} .
$$

When $r \rightarrow r_{H}$, we have

$$
\begin{aligned}
\left.\omega^{ \pm}\right|_{r \rightarrow r_{H}}= & \omega_{0} \\
= & \left.k_{\theta} \frac{g^{12}-g^{j 2} r_{H, j}}{g^{01}-g^{0 j} r_{H, j}}\right|_{r \rightarrow r_{H}}+\left.k_{\varphi} \frac{g^{13}-g^{j 3} r_{H, j}}{g^{01}-g^{0 j} r_{H, j}}\right|_{r \rightarrow r_{H}} \\
& +\left.e \frac{A_{\mu} g^{\mu 1}-A_{\mu} g^{\mu j} r_{H, j}}{g^{01}-g^{0 j} r_{H, j}}\right|_{r \rightarrow r_{H}} .
\end{aligned}
$$

$\omega_{0}$ is the maximum value of negative energy state after overlapping of positive and negative energy levels near the event horizon. Therefore, the incident negative energy particles within the event horizon satisfying $\mu_{0}<\omega \leq \omega_{0}$ will become emerging positive energy particles via quantum tunneling effect. This is the nonthermal radiative process that has no relation to the temperature.

\section{Discussion}

The Hamilton-Jacobi equation describes the general behavior of moving particles in a nonstatistical way, so it is suitable for investigating the nonthermal radiation. 
The thermal radiation spectrum (33) demonstrates that the total interaction energy of Dirac particles in a general nonstationary black hole is

$$
\begin{aligned}
\omega_{0}= & \left.k_{\theta} \frac{g^{12}-g^{j 2} r_{H, j}}{g^{01}-g^{0 j} r_{H, j}}\right|_{r \rightarrow r_{H}}+\left.k_{\varphi} \frac{g^{13}-g^{j 3} r_{H, j}}{g^{01}-g^{0 j} r_{H, j}}\right|_{r \rightarrow r_{H}} \\
& +\left.e \frac{A_{\mu} g^{\mu 1}-A_{\mu} g^{\mu j} r_{H, j}}{g^{01}-g^{0 j} r_{H, j}}\right|_{r \rightarrow r_{H}}+C_{1},
\end{aligned}
$$

where

$$
\begin{aligned}
& C_{1}=\left.\frac{1}{g^{01}-g^{0 j} r_{H, j}}\right|_{r \rightarrow r_{H}} \\
& \times\left\{\left\{\frac{[(\bar{\mu}-\bar{\gamma})-(\mu-\gamma)] l^{1}}{2 i}+\frac{[(\varepsilon-\rho)-(\bar{\varepsilon}-\bar{\rho})] n^{1}}{2 i}\right.\right. \\
& \left.-\frac{\left(\bar{m}^{\mu} m_{, \mu}^{1}-m^{\mu} \bar{m}_{, \mu}^{1}\right)}{2 i}\right\} \\
& -\left\{\frac{[(\bar{\mu}-\bar{\gamma})-(\mu-\gamma)] l^{j}}{2 i}+\frac{[(\varepsilon-\rho)-(\bar{\varepsilon}-\bar{\rho})] n^{j}}{2 i}\right. \\
& \left.\left.-\frac{\left(\bar{m}^{\mu} m_{, \mu}^{j}-m^{\mu} \bar{m}_{, \mu}^{j}\right)}{2 i}\right\} r_{H, j}\right\}\left.\right|_{r \rightarrow r_{H}} \\
& +\left.\frac{1}{\left(g^{01}-g^{0 j} r_{H, j}\right)\left(n^{j} r_{H, j}-n^{1}\right)}\right|_{r \rightarrow r_{H}} \\
& \times \frac{1}{2 i}\left\{\left\{\left[n^{\mu} \bar{m}_{, \mu}^{1}-\bar{m}^{\mu} n_{, \mu}^{1}+(\pi-\alpha) n^{1}+(\bar{\mu}-\bar{\gamma}) \bar{m}^{1}\right.\right.\right. \\
& \left.-(\mu-\gamma) \bar{m}^{1}-(\bar{\beta}-\bar{\tau}) n^{1}\right] \\
& -\left[n^{\mu} \bar{m}_{, \mu}^{j}-\bar{m}^{\mu} n_{, \mu}^{j}+(\pi-\alpha) n^{j}+(\bar{\mu}-\bar{\gamma}) \bar{m}^{j}\right. \\
& \left.-(\mu-\gamma) \bar{m}^{j}-(\bar{\beta}-\bar{\tau}) n^{j}\right] \\
& \left.\times r_{H, j}\right\}\left(m^{1}-m^{j} r_{H, j}\right) \\
& -\left\{\left[n^{\mu} m_{, \mu}^{1}-m^{\mu} n_{, \mu}^{1}\right.\right. \\
& +(\bar{\pi}-\bar{\alpha}) n^{1}+(\mu-\gamma) m^{1}-(\bar{\mu}-\bar{\gamma}) m^{1} \\
& \left.-(\beta-\tau) n^{1}\right]-\left[n^{\mu} m_{, \mu}^{j}-m^{\mu} n_{, \mu}^{j}+(\bar{\pi}-\bar{\alpha}) n^{j}\right. \\
& +(\mu-\gamma) m^{j}-(\bar{\mu}-\bar{\gamma}) m^{j} \\
& \left.\left.-(\beta-\tau) n^{j}\right] r_{H, j}\right\} \\
& \left.\times\left(\bar{m}^{1}-\bar{m}^{j} r_{H, j}\right)\right\}\left.\right|_{r \rightarrow r_{H}},
\end{aligned}
$$

for $\Psi=F_{1}$, and

$$
\begin{aligned}
C_{1}= & \left.\frac{1}{g^{01}-g^{0 j} r_{H, j}}\right|_{r \rightarrow r_{H}} \\
& \times\left\{\left\{\frac{[(\mu-\gamma)-(\bar{\mu}-\bar{\gamma})] l^{1}}{2 i}\right.\right. \\
& \left.+\frac{[(\bar{\varepsilon}-\bar{\rho})-(\varepsilon-\rho)] n^{1}}{2 i}-\frac{\left(m^{\mu} \bar{m}_{, \mu}^{1}-\bar{m}^{\mu} m_{, \mu}^{1}\right)}{2 i}\right\}
\end{aligned}
$$$$
-\left\{\frac{[(\mu-\gamma)-(\bar{\mu}-\bar{\gamma})] l^{j}}{2 i}+\frac{[(\bar{\varepsilon}-\bar{\rho})-(\varepsilon-\rho)] n^{j}}{2 i}\right.
$$$$
\left.\left.-\frac{\left(m^{\mu} \bar{m}_{, \mu}^{j}-\bar{m}^{\mu} m_{, \mu}^{j}\right)}{2 i}\right\} r_{H, j}\right\}\left.\right|_{r \rightarrow r_{H}}
$$

$$
+\left.\frac{1}{\left(g^{01}-g^{0 j} r_{H, j}\right)\left(l^{j} r_{H, j}-l^{1}\right)}\right|_{r \rightarrow r_{H}}
$$$$
\times \frac{1}{2 i}\left\{\left\{\left[l^{\mu} m_{, \mu}^{1}-m^{\mu} l_{, \mu}^{1}+(\beta-\tau) l^{1}\right.\right.\right.
$$$$
\left.+(\bar{\varepsilon}-\bar{\rho}) m^{1}-(\varepsilon-\rho) m^{1}-(\bar{\pi}-\bar{\alpha}) l^{1}\right]
$$$$
-\left[l^{\mu} m_{, \mu}^{j}-m^{\mu} l_{, \mu}^{j}+(\beta-\tau) l^{j}+(\bar{\varepsilon}-\bar{\rho}) m^{j}\right.
$$$$
\left.\left.-(\varepsilon-\rho) m^{j}-(\bar{\pi}-\bar{\alpha}) l^{j}\right] r_{H, j}\right\}
$$$$
\times\left(\bar{m}^{1}-\bar{m}^{j} r_{H, j}\right)
$$$$
-\left\{\left[l^{\mu} \bar{m}_{, \mu}^{1}-\bar{m}^{\mu} l_{, \mu}^{1}+(\bar{\beta}-\bar{\tau}) l^{1}+(\varepsilon-\rho) \bar{m}^{1}\right.\right.
$$$$
\left.-(\bar{\varepsilon}-\bar{\rho}) \bar{m}^{1}-(\pi-\alpha) l^{1}\right]
$$$$
-\left[l^{\mu} \bar{m}_{, \mu}^{j}-\bar{m}^{\mu} l_{, \mu}^{j}+(\bar{\beta}-\bar{\tau}) l^{j}\right.
$$$$
\left.\left.+(\varepsilon-\rho) \bar{m}^{j}-(\bar{\varepsilon}-\bar{\rho}) \bar{m}^{j}-(\pi-\alpha) l^{j}\right] r_{H, j}\right\}
$$$$
\left.\times\left(m^{1}-m^{j} r_{H, j}\right)\right\}\left.\right|_{r \rightarrow r_{H}},
$$

for $\Psi=F_{2}$.

This chemical potential $\omega_{0}$ is composed of three parts. The sum of the first two terms $k_{\theta}\left(\left(g^{12}-g^{j 2} r_{H, j}\right) /\left(g^{01}-\right.\right.$ $\left.\left.g^{0 j} r_{H, j}\right)\right)\left.\right|_{r \rightarrow r_{H}}+\left.k_{\varphi}\left(\left(g^{13}-g^{j 3} r_{H, j}\right) /\left(g^{01}-g^{0 j} r_{H, j}\right)\right)\right|_{r \rightarrow r_{H}}$ is the rotational energy arising from the coupling between different components of generalized momentum of particles and different rotations of the black hole. The second part is the electromagnetic interaction energy $e\left(\left(A_{\mu} g^{\mu 1}-\right.\right.$ $\left.\left.A_{\mu} g^{\mu j} r_{H, j}\right) /\left(g^{01}-g^{0 j} r_{H, j}\right)\right)\left.\right|_{r \rightarrow r_{H}}$. The third part $C_{1}$ (which characterizes a new extra coupling effect arising from the interaction between the intrinsic spin of particles and the generalized momentum of the evaporating black hole and 
vanishes in the case of scalar particles (we will see it later) and has no classical correspondence) gives a general expression of the new extra spin-rotation coupling and the spin-acceleration coupling effect. When considering concrete black holes like those of [18-21], $C_{1}$ will degenerate and represent the concrete extra coupling effects in [18-21].

Now we consider the asymptotic behavior of the minimally electromagnetic coupling Klein-Gordon equation near the event horizon. The explicit form of wave equation describing the dynamic behavior of scalar particles with mass $\mu_{0}$ and charge $e$ is as follows:

$$
\begin{aligned}
& \frac{1}{\sqrt{-g}}\left[\left(\frac{\partial}{\partial x^{\mu}}+i e A_{\mu}\right) \sqrt{-g} g^{\mu \nu}\left(\frac{\partial}{\partial x^{\nu}}+i e A_{\nu}\right)\right] \\
& \quad \times \phi(x)-\mu_{0}^{2} \phi(x)=0 .
\end{aligned}
$$

Applying the same method in Section 2 to (43), throughout long calculations, one can get a similar form of (22) near the event horizon, except that the coefficient $A=2 i C_{0}+C_{3}+C_{4}^{\prime}$, where

$$
\begin{aligned}
C_{4}^{\prime}=( & \left(\left(g_{, \mu}^{\mu 1}+(1 / \sqrt{-g}) g^{\mu 1}\left(\partial \sqrt{-g} / \partial x^{\mu}\right)\right)\right. \\
& \left.-\left(g_{, \mu}^{\mu j}+(1 / \sqrt{-g}) g^{\mu j}\left(\partial \sqrt{-g} / \partial x^{\mu}\right)\right) r_{H, j}-g^{j k} r_{H, j k}\right) \\
& \left.\times\left(g^{01}-g^{0 j} r_{H, j}\right)^{-1}\right)\left.\right|_{r \rightarrow r_{H}},
\end{aligned}
$$

and the chemical potential

$$
\begin{aligned}
\omega_{0}= & k_{\theta} \frac{B}{2}+k_{\varphi} \frac{C}{2}+\frac{\operatorname{Im}(A)}{2} \\
= & \left.k_{\theta} \frac{g^{12}-g^{j 2} r_{H, j}}{g^{01}-g^{0 j} r_{H, j}}\right|_{r \rightarrow r_{H}}+\left.k_{\varphi} \frac{g^{13}-g^{j 3} r_{H, j}}{g^{01}-g^{0 j} r_{H, j}}\right|_{r \rightarrow r_{H}} \\
& +\left.e \frac{A_{\mu} g^{\mu 1}-A_{\mu} g^{\mu j} r_{H, j}}{g^{01}-g^{0 j} r_{H, j}}\right|_{r \rightarrow r_{H}} .
\end{aligned}
$$

One can see that $C_{1}=0$ in this case. Furthermore, it is interesting to find that (45) and (39) are the same, which means that the chemical potential in thermal radiation spectrum is equal to the highest energy of negative energy state of scalar particles in nonthermal radiation for general nonstationary black holes. This is an important conclusion that reveals the relationship between the two kinds of radiative processes of black holes, which was ignored before. Equation (39) is derived from Section 3 by using the Hamilton-Jacobi equation which applies to both fermion and boson. So (39) is also for scalar particles. This is why we can compare these two equations.

On the other hand, because, in physics, a general physical process should satisfy quantitative causal relation with noloss-no-gain character [32-34], for example, [35] uses the noloss-no-gain homeomorphic map transformation satisfying the quantitative causal relation to gain exact strain tensor formulas in Weitzenböck manifold. In fact, some changes (cause) of some quantities in (7) must result in the relative some changes (result) of the other quantities in (7) so that (7)'s right hand side keeps no-loss-no-gain, that is, zero; namely, (6) also satisfies the quantitative causal relation with no-loss-no-gain character. And (2), (7)-(11), (37) and (43) also satisfy the quantitative causal relation with no-loss-nogain character in the same way. Hence, the investigations in this paper are consistent. Also, the investigations of this paper provide an alternative and convenient way to obtain the Hawking temperature and are the generalizations and developments of the investigations of $[42,43]$ about quantum radiation of general nonstationary black holes.

\section{Summary and Conclusion}

This paper carefully investigates quantum radiation properties of Dirac particles in general nonstationary black holes, generally shows that the temperature and the shape of the event horizon of black holes depend on both the time and different angles in general condition, and further gives a general expression of the new extra coupling effect arising from the interaction between the intrinsic spin of particles and the generalized momentum of the general nonstationary black hole. Finally, this paper shows that the new extra coupling effect is absent from the thermal radiation spectrum in this case; this paper generally reveals a relationship that is ignored before between thermal radiation and nonthermal radiation of black holes in detail, which is that the chemical potential in thermal radiation spectrum is equal to the highest energy of the negative energy state in nonthermal radiation for general nonstationary black holes.

\section{Conflict of Interests}

The authors declare that there is no conflict of interests regarding the publication of this paper.

\section{Acknowledgments}

The authors are grateful for Professor R. G. Cai and Professor Z. Zhao for their useful discussions and comments. The work is supported by the Project of Knowledge Innovation Program (PKIP) of the Chinese Academy of Sciences (no. KJCX2.YW.W10) National Natural Science Foundation of China (no. 11275017 and no. 11173028).

\section{References}

[1] S. W. Hawking, "Black hole explosions?" Nature, vol. 284, pp. 30-31, 1974.

[2] S. W. Hawking, "Particle creation by black holes," Communications in Mathematical Physics, vol. 43, no. 3, pp. 199-220, 1975.

[3] B. Hartle and S. W. Hawking, "Path-integral derivation of blackhole radiance," Physical Review D, vol. 13, no. 8, pp. 2188-2203, 1976.

[4] R. M. Wald, "On particle creation by black holes," Communications in Mathematical Physics, vol. 45, p. 9, 1975.

[5] W. G. Unruh, "Notes on black hole evaporation," Physical Review D, vol. 14, pp. 870-892, 1976. 
[6] T. Damour and R. Ruffini, "Black hole evaporation in the KleinSauter-Heisenberg-Euler formalism," Physical Review D, vol. 14, pp. 332-334, 1976.

[7] S. Sannan, "Heuristic derivation of the probability distributions of particles emitted by a black hole," General Relativity and Gravitation, vol. 20, no. 3, pp. 239-246, 1988.

[8] Z. Zhao and X. X. Dai, "A new method dealing with Hawking effects of evaporating black holes," Modern Physics Letters A, vol. 7, no. 20, pp. 1771-1778, 1992.

[9] C. G. Huang, L. Liu, and Z. Zhao, "The thermodynamical approach to the back reaction problem," General Relativity and Gravitation, vol. 25, no. 12, pp. 1267-1275, 1993.

[10] Z. Zhao, C. Q. Yang, and Q. A. Ren, "Hawking effect in Vaidyade Sitter space-time," General Relativity and Gravitation, vol. 26, no. 11, pp. 1055-1065, 1994.

[11] W. A. Hiscock, "Models of evaporating black holes. II. effects of the outgoing created radiation," Physical Review D, vol. 23, no. 12, pp. 2823-2827, 1981.

[12] R. Balbinot, "Back reaction and the small-mass regime," Physical Review D, vol. 33, no. 6, pp. 1611-1615, 1986.

[13] M. C. Sun, R. Zhao, and Z. Zhao, "Hawking effect of a rotating, arbitrarily accelerating black hole," Il Nuovo Cimento B, vol. 110, no. 7, pp. 829-837, 1995.

[14] S. Q. Wu and X. Cai, "Exact solutions to sourceless charged massive scalar field equation on Kerr-Newman background," Journal of Mathematical Physics, vol. 40, pp. 4538-4548, 1999.

[15] S. Q. Wu and X. Cai, "Massive complex scalar field in the KerrSen geometry: exact solution of wave equation and Hawking radiation," Journal of Mathematical Physics, vol. 44, pp. 10841088, 2003.

[16] S. Chandrasekhar, The Mathematical Theory of Black Holes, Oxford University Press, New York, NY, USA, 1983.

[17] D. Page, "Dirac equation around a charged, rotating black hole," Physical Review D, vol. 14, no. 6, pp. 1509-1510, 1976.

[18] S. Q. Wu and X. Cai, "Hawking radiation of dirac particles in a Variable-mass Kerr space-time," General Relativity and Gravitation, vol. 33, no. 7, pp. 1181-1195, 2001.

[19] S. Q. Wu and X. Cai, "Addendum: Hawking radiation of photons in a Variable-mass Kerr black hole," General Relativity and Gravitation, vol. 34, no. 4, pp. 557-564, 2002.

[20] S. Q. Wu and X. Cai, "Hawking radiation of a non-stationary Kerr-Newman black hole: spin-rotation coupling effect," General Relativity and Gravitation, vol. 34, no. 5, pp. 605-617, 2002.

[21] S. Q. Wu and X. Cai, "Hawking radiation of dirac particles in an arbitrarily accelerating Kinnersley black hole," General Relativity and Gravitation, vol. 34, no. 8, pp. 1207-1220, 2002.

[22] R. G. Cai, "A note on thermodynamics of black holes in Lovelock gravity," Physics Letters B, vol. 582, no. 3-4, pp. 237242, 2004.

[23] R. G. Cai, "Gauss-Bonnet black holes in AdS spaces," Physical Review D, vol. 65, no. 8, Article ID 084014, 9 pages, 2002.

[24] R. G. Cai, "Cardy-Verlinde formula and thermodynamics of black holes in de Sitter spaces," Nuclear Physics B, vol. 628, no. 1-2, pp. 375-386, 2002.

[25] B. Carter, "Global structure of the Kerr family of gravitational fields," Physical Review, vol. 174, no. 5, pp. 1559-1571, 1968.

[26] W. G. Unruh, "Second quantization in the Kerr metric," Physical Review D, vol. 10, no. 10, pp. 3194-3205, 1974.

[27] N. Deruelle and R. Ruffini, "Quantum and classical relativistic energy states in stationary geometries," Physics Letters B, vol. 52, no. 4, pp. 437-441, 1974.
[28] T. Damour and N. Deruelle, "Klein paradox and vacuum polarization," in Proceedings of the 1st Marcel Grossmann Meeting on General Relativity, pp. 476-483, North-Holland Publishing, Trieste, Italy, 1975.

[29] S. W. Kim, "Black hole radiation in the Vaidya metric," Physics Letters A, vol. 141, no. 5-6, pp. 238-242, 1989.

[30] R. Balbinot and A. Barletta, "The backreaction and the evolution of quantum black holes," Classical and Quantum Gravity, vol. 6, no. 2, p. 195, 1989.

[31] M. H. Ali, "Letter: quantum nonthermal radiation of nonstationary Kerr-Newman-de Sitter black holes," General Relativity and Gravitation, vol. 36, no. 5, pp. 1171-1181, 2004.

[32] E. Newman and R. Penrose, "An approach to gravitational radiation by a method of spin coefficients," Journal of Mathematical Physics, vol. 3, no. 3, p. 566, 1962.

[33] M. E. Ortiz and F. Vendrell, "Thermal radiation in nonstatic curved spacetimes: quantum mechanical path integrals and configuration space topology," Physical Review D, vol. 59, no. 8, Article ID 084005, 8 pages, 1999.

[34] D. Christodoulou and R. Ruffini, "Reversible transformations of a charged black hole," Physical Review D, vol. 4, no. 12, pp. 3552-3555, 1971.

[35] Y. C. Huang, X. G. Lee, and M. X. Shao, "Unified expressions of all integral variational principles," Modern Physics Letters, vol. 21, no. 14, pp. 1107-115, 2006.

[36] Y. C. Huang and Q. H. Huo, "Faddeev-Senjanovic quantization of $\mathrm{SU}(n) N=2$ supersymmetric gauge field system with a nonAbelian Chern-Simons topological term and its fractional spin," Physics Letters B, vol. 662, no. 3, pp. 290-296, 2008.

[37] Y. C. Huang and C. X. Yu, "Quantization and spectrum of an open 2-brane," Physical Review D, vol. 75, no. 4, Article ID 044011, 7 pages, 2007.

[38] L. Liao and Y. C. Huang, "Non-equivalence of Faddeev-Jackiw method and Dirac-Bergmann algorithm and the modification of Faddeev-Jackiw method for keeping the equivalence," Annals of Physics, vol. 322, no. 10, pp. 2469-2484, 2007.

[39] Y. C. Huang and G. Weng, "Solution of Wheeler-De Witt equation, potential well and tunnel effect," Communications in Theoretical Physics, vol. 44, no. 4, pp. 757-761, 2005.

[40] Y. C. Huang, L. Liao, and X. G. Lee, "Faddeev-Jackiw canonical path integral quantization for a general scenario, its proper vertices and generating functional," The European Physical Journal C, vol. 60, no. 3, pp. 481-487, 2009.

[41] Y. C. Huang and B. L. Lin, "General Weitzenböck theory of crystals with different dislocation distributions," Physics Letters A, vol. 299, no. 5-6, pp. 644-649, 2002.

[42] J. C. Hua and Y. C. Huang, "Characteristics of quantum radiation of slowly varying nonstationary Kerr-Newman black holes," International Journal of Modern Physics A, vol. 24, no. 10, p. 1889, 2009.

[43] J. C. Hua and Y. C. Huang, "Quantum radiation of general nonstationary black holes," Europhysics Letters, vol. 85, no. 3, Article ID 30007, 2009. 

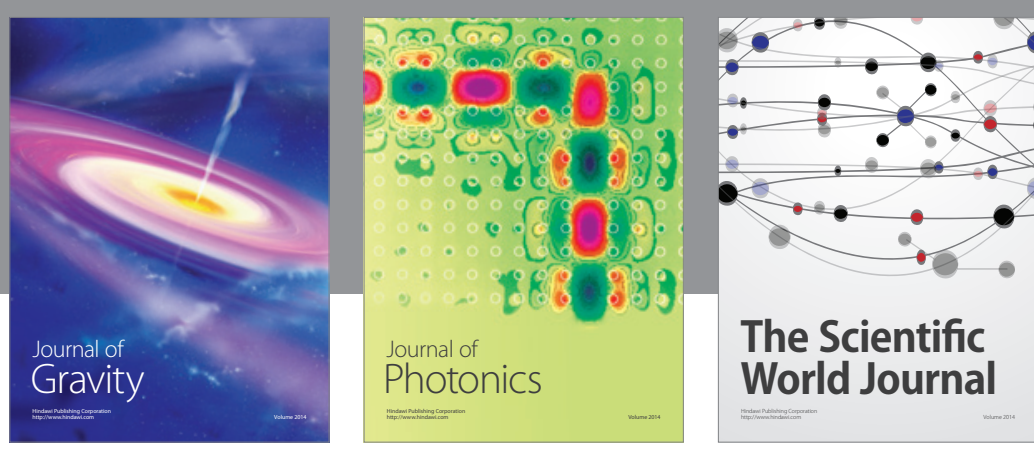

The Scientific World Journal
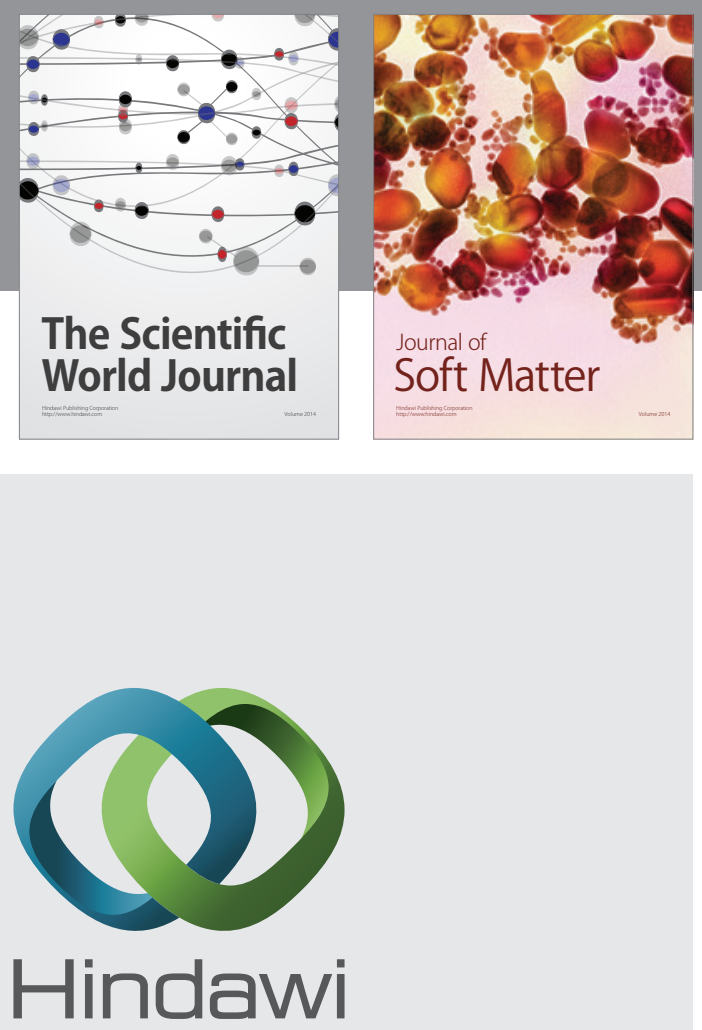

Submit your manuscripts at

http://www.hindawi.com

nternational Journal of

Statistical Mechanics
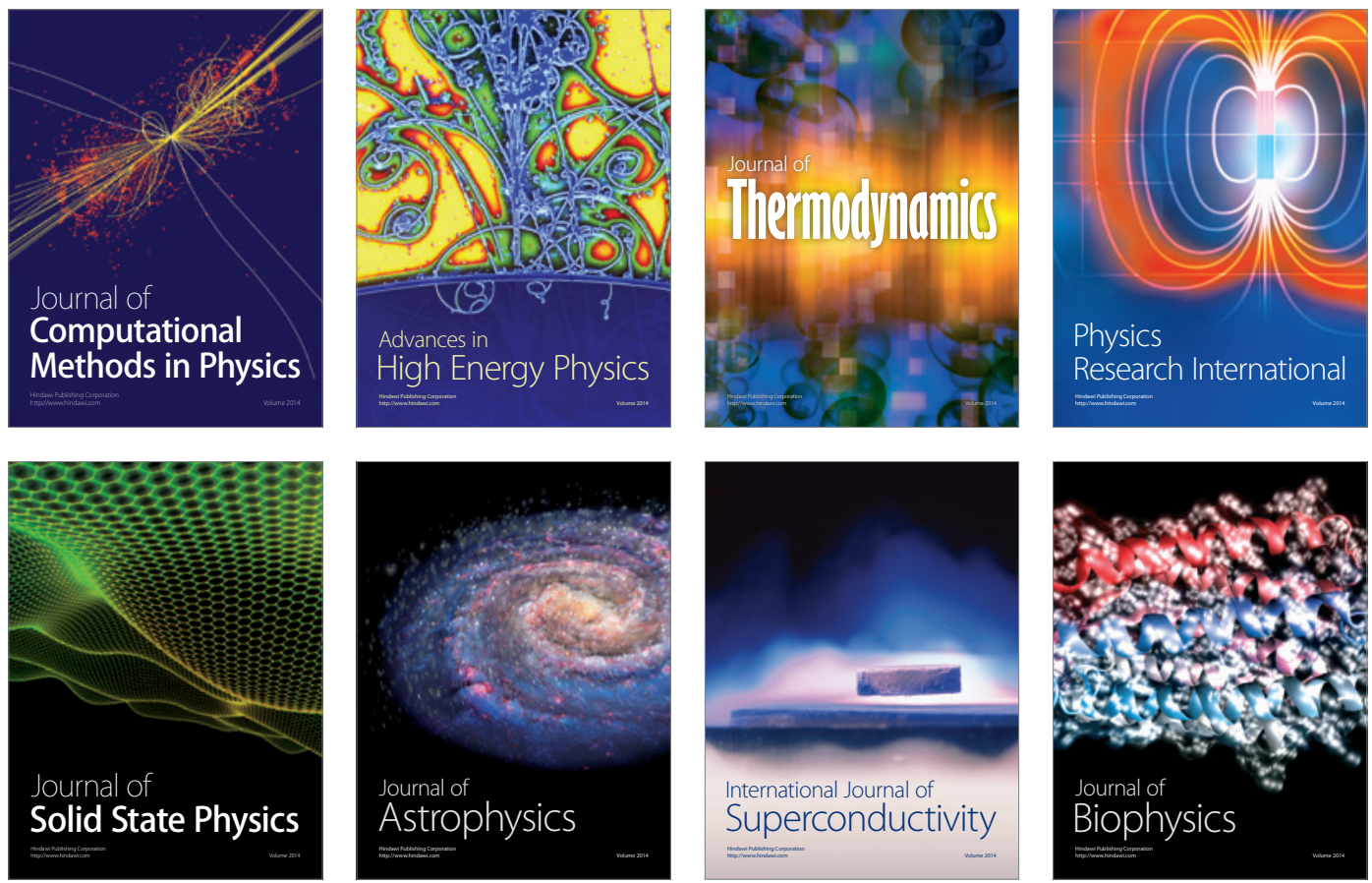
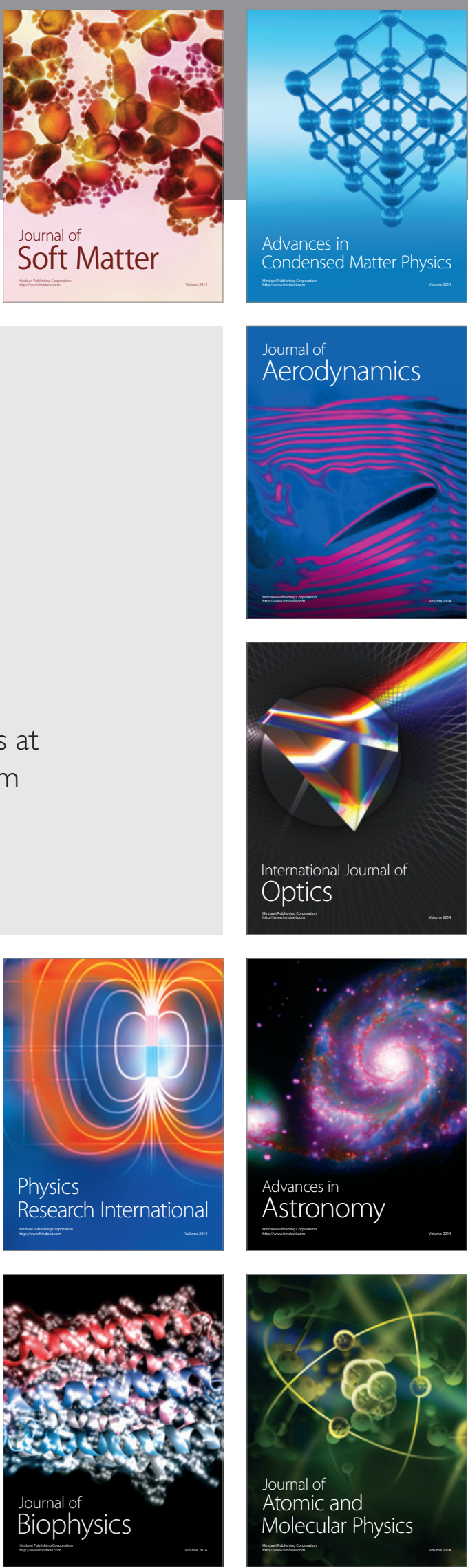